\title{
Does the Antimalarial Drug Quinine Contribute to Muscle Fibro/Adipogenic Progenitor Fibrogenesis?
}

\author{
J. H. Jay', M. Liư', X. Liu²,3, B. T. Feeley2,3, C. S. Sabatini ${ }^{2,4}$ \\ 1 Alabama College of Osteopathic Medicine, Dothan (AL), U.S.A. \\ 2 Department of Orthopaedic Surgery, University of California San Francisco, San Francisco (CA), U.S.A. \\ 3 San Francisco Veterans Affairs Medical Center, San Francisco (CA), U.S.A. \\ ${ }^{4}$ Division of Orthopaedic Surgery, UCSF Benioff Children's Hospital Oakland, Oakland (CA), U.S.A.
}

\section{CORRESPONDING AUTHOR:}

Coleen S. Sabatini

Division of Orthopaedic Surgery

UCSF Benioff Children's Hospital Oakland

$74752^{\text {nd }}$ Street

Oakland (CA) 94609, U.S.A

E-mail: coleen.sabatini@ucsf.edu

DOI:

10.32098/mltj.04.2021.04

LEVEL OF EVIDENCE: 3

\begin{abstract}
SUMMARY
Background. In many resource-limited countries, children are routinely given intramuscular (IM) injections of medication to treat pain or illness. IM injections are suspected in the development of gluteal fibrosis (GF) in children, a condition that limits normal hip motion and function. The mechanism by which GF develops is not understood. Our study examines a commonly IM administered antimalarial in Uganda, quinine, to assess its ability to cause fibrogenesis of muscle fibro/adipogenic progenitors (FAPs), the cellular source of muscle fibrosis. The purpose was to evaluate if quinine itself could alter muscle cells and cause fibrosis.

Methods. FAPs were isolated from skeletal muscle in wildtype C57BL/6 mouse with florescence-activated cell sorting (FACS). After sorting, the FAPs were cultured in standard media until they reached $80 \%$ confluence. The cells were then cultured in a series of quinine concentrations. Fibrogenesis of FAPs was determined with RT-qPCR of fibrogenic markers.

Results. The RT-PCR results showed increased $\alpha \mathrm{SMA}$, vimentin and collagen-1 expression in quinine exposed cells. At lower quinine dosages, expression increased in stemness markers; Sox2, cMyc, Oct-4, and Nanog. Conversely, at higher dosages quinine decreased stemness marker expression. Lastly, quinine increased TGF $\beta 1$ and Ki67 and decreased BMP7.

Conclusions. Our findings suggest that quinine induces FAP fibrogenesis and reduces FAP stemness. Further study is needed, but if confirmed that Quinine induces fibrogenesis, limiting the use of Quinine may be an effective public health intervention to reduce cases of gluteal fibrosis and resultant childhood disability.
\end{abstract}

KEY WORDS

Fibro/adipogenic progenitors; gluteal fibrosis; intramuscular injections; TGFß1; quinine.

\section{BACKGROUND}

In many resource-limited countries, children are routinely given intramuscular (IM) injections for the delivery of medication to treat febrile illnesses/communicable diseases (1-10). IM injections are thought to be the underlying cause of gluteal fibrosis (GF) - a fibrotic infiltration of the gluteal muscles that results in a loss of flexion and adduction of the hips (1,2, 4-6). The resultant abduction contracture of the hips impacts children's ability to sit and squat normally. Additionally, this condition limits their ability to attend school and carry out normal daily activities such as using the toilet and performing chores. In certain regions of Uganda, such as the District of Kumi, there are many children with this condition - with as many as $30 \%$ of visits to a musculoskeletal clinic and $40 \%$ of outreach visits being for an injection related injury $(1,4)$. In this particular region of Eastern Uganda, some of the local medical providers suspect that quinine is the culprit in their high number of GF cases. Studies have indicated that over $80 \%$ of children affected by GF had received multiple IM quinine injections prior to their post injection complications 
$(1,4)$. However, the exact mechanism of how GF develops through the administration of IM injections is still not understood. As such, we have sought to investigate the basic science aspects of GF in order to better understand this condition and impact future prevention and treatment efforts.

Our study examines a commonly IM administered antimalarial in Uganda, quinine, to assess its ability to cause fibrotic changes in skeletal muscle cells $(1,2,4,5,7-9,11,12)$. We studied quinine in particular because, through the senior author's (CS) work on GF in Uganda, quinine has been anecdotally implicated as the main agent in inducing GF. Should it induce changes at the cellular level, this can further our understanding of the mechanism by which GF develops in this population. Using a cell model, we exposed fibrogenic/ adipogenic progenitor (FAPs) cells in culture media conditions to different concentrations of quinine in order to determine its effects on FAP fibrogenic differentiation and filament production $(4,11,13-16,18)$. This investigation allowed us to begin to uncover the microscopic events that potentially lead to macroscopic muscle fibrosis and loss of function (4).

FAP's are integral regulators of satellite cells (skeletal muscle stem cells) in acute injury states $(14,15)$. During times of cellular stress, FAP's assimilate environmental conditions into signals that regulate satellite cell activities $(14,15)$. Interestingly, the abnormal activity of FAP's has been linked to the development of pathologic states within skeletal muscle such as impaired muscle regeneration and fibrosis $(14,15)$. We anticipate that quinine will significantly alter FAP gene expression leading to the abnormal healing of skeletal muscle and ultimately resulting in fibrosis.

\section{MATERIALS AND METHODS}

\section{FAP sorting and isolation}

In order to study the effects of quinine on FAP differentiation and filament production, we isolated the cell population of CD31; CD45, Integrina $7^{-}, \mathrm{Sca}-1^{+}$and PDGFR $\alpha^{+}$ FAPs from skeletal muscle of 3-month-old male wildtype C57BL/6 mice (Jackson laboratory Corp., Sacramento, CA). After C57BL/6 mice were sacrificed, skeletal muscles were harvested and minced into $1 \mathrm{~mm}$ chunks with sterile scissors in cell culture hood. We then incubated the minced skeletal muscle with $0.2 \%$ Collagenase II for 90 minutes in a 37 ${ }^{\circ} \mathrm{C}$ sterile water bath. Next, forty milliliter washing buffer (F/10, 10\% Horse Serum, $1 \times$ HEPES) was added into the mixture and then centrifuged at $1500 \mathrm{rpm}$ for 5 minutes at room temperature. The supernatant was then transferred to a new $50 \mathrm{~mL}$ centrifuge tube and set aside. The remnants were then rinsed with washing buffer and spun down for 5 minutes at $1500 \mathrm{rpm}$. The supernatant then collected and combined with the supernatant from the first round of centrifuging. Then D2 solution $(0.06 \%$ Collagenase II, $0.15 \%$ Dispase with washing buffer) was added to the combined supernatant and incubated at $37^{\circ} \mathrm{C}$ for 30 minutes. This solution was then passed through a $70 \mu \mathrm{m}$ cell strainer (VWR International) and then a $40 \mu \mathrm{m}$ cell strainer (VWR International). The filtered cells were collected and washed with $40 \mathrm{~mL}$ FACS buffer $(2.5 \%$ FBS, $20 \mathrm{mM}$ EDTA, $1 \times$ PBS $)$ and centrifuged at $1500 \mathrm{rpm}$ for 5 minutes. The filtered cell supernatant was discarded, and the cell pellets were re-suspended with 500 $\mu \mathrm{L}$ of FACS buffer. The cell solution was then incubated with anti-CD31-FITC (BD bioscience), anti-CD45-FITIC (BD bioscience), anti-integrin $\alpha 7$-APC (R\&D systems) PE-Cy7Sca1 (BD bioscience, Clone. E13-161.7) and anti-cd140a(PDGFR $\alpha$ )-BV421 (BD bioscience, Clone APA5) for 30 minutes before being sorted with FACSAria ${ }^{\mathrm{TM}}$ II sorter (BD biosciences). FAPs were collected as the CD31/CD45-/Integrin $\alpha 7^{-} / \mathrm{Sca}-1^{+} / \mathrm{PDGFR}^{+}$cell population. The SFVAMC Institutional Animal Care and Use Committee (IACUC) approved all procedures and handling of the animals.

\section{Cell culture}

24 well plates were coated with 1\% Matrigel in DMEM for 1 hour at room temperature before cell seeding. After sorting, the FAPs were seeded into Matrigel pre-coated 24 well plates and at a density of 5,000 cells per well. Cells were cultured for one week with standard cell culture medium (Ham's F-10, 10\% fetal bovine serum, $10 \mathrm{ng} / \mathrm{mL} \beta \mathrm{FGF}$ and $1 \%$ antibiotic-antimycotic solution, Thermo Fisher Scientific, MA USA). Then one 24 well plate of cells was set aside to be used as our negative control. The rest of the cells were then cultured in $0.5 \mathrm{~mL}$ of standard culture media with the addition of a fixed concentration of serially diluted quinine for two weeks before processing for RT-qPCR. Using a logarithmic serial dilution, we exposed the cells to quinine's injection dosage concentration (IDC), the concentration that quinine is injected into the patient, and successive diluted concentration until we ended at one thousand times below the IDC $(\mathrm{IDC}, \mathrm{IDC} \times 0.1, \mathrm{IDC} \times 0.01, \mathrm{IDC} \times 0.001)(1,3,4,8,9,12$, $17,18)$. Lastly, our negative control group was exposed to $0.5 \mathrm{~mL}$ of standard culture media for 2 weeks. We also tested the effect of quinine on the cells at its peak serum concentration (SC), the highest concentration of quinine found in the blood stream after administration $(12,17,18)$. Six biological replicates per condition were run.

\section{Real Time qPCR}

Total RNA was extracted from the cells on day 14 using a Trizol reagent (Applied Biosystems) according to the 
manufacturer's instructions. Real-time qPCR was run with Fast SYBR Green Master Mix (Applied Biosystems) on a Viia7 Real Time Detection System (Applied Biosyste$\mathrm{ms})$. This test was performed to quantify the expression of TGF $\beta-1$, BMP7, and Ki67, the fibrotic markers collagen-1, vimentin, and $\alpha$ SMA expression and the stemness markers Nanog, Sox 2 , Oct-4 and cMyc $(13,14)$. The $\Delta \Delta$ Ct method was used to compare gene expression between the different quinine concentrations using GAPDH as the housekeeping gene.

\section{Statistical analysis}

For all analyses, ANOVA was used to assess for significance. Significance was defined as $p<0.05$. Data are presented as mean \pm standard error of measurement.

\section{Ethical standards}

Our study meets the ethical standards of Muscle Ligaments and Tendons Journal. From our cell line development and authentication to the reporting of our molecular results obtained through basic science practices $(19,20)$.

\section{RESULTS}

\section{Quinine induces fibrogenesis}

The RT-PCR results showed an increase in $\alpha \mathrm{SMA}$ expression (+ 3.02-fold average, $\mathrm{p}<.05, \mathrm{SE}=.46)$ and vimentin $(+81.89$-fold average, $\mathrm{p}<.05, \mathrm{SE}=3.89)$ across all quinine concentrations - except IDC - at the end of the 14 days

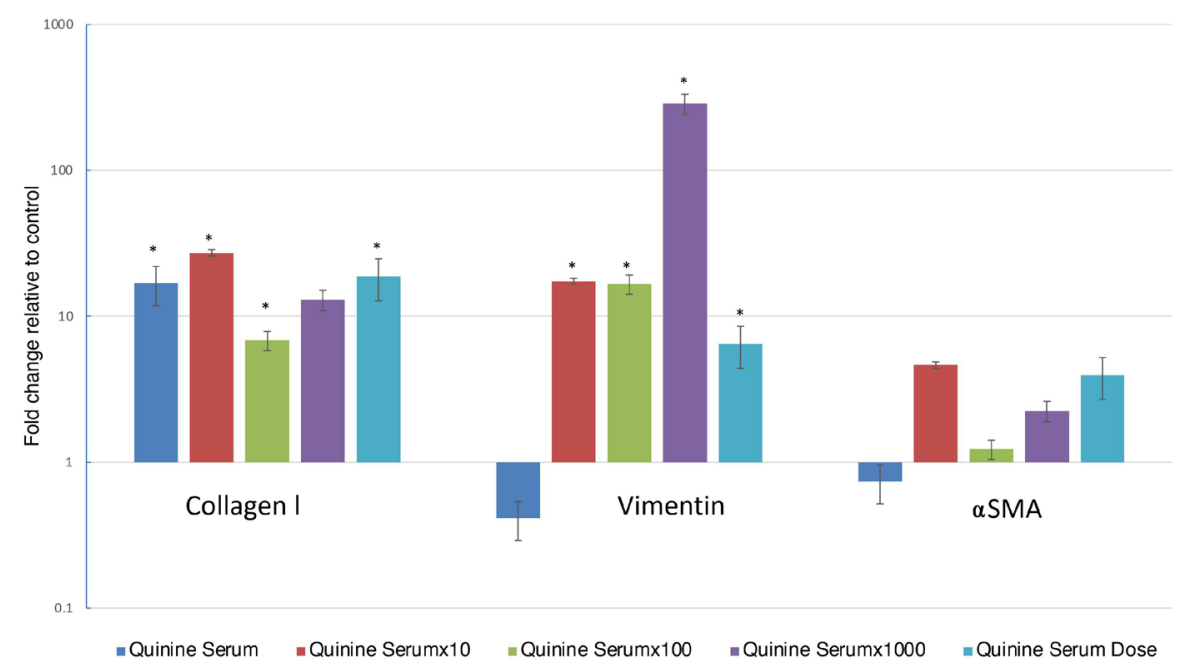

Figure 1. Quinine increases fibrogenesis marker expression significantly in FAPs. compared to the control (Graph 1). Similarly, there was a significant average fold increase in collagen-1 $(+16.55$-fold average, $\mathrm{p}<.05, \mathrm{SE}=1.22$ ) across all quinine concentrations (figure 1).

\section{Quinine reduces stemness}

Furthermore, at lower dosages quinine increased stemness markers in Sox2 $(+3.57$-fold, $\mathrm{p}<.05, \mathrm{SE}=.3528)$, cMyc $(+7.38$-fold, $\mathrm{p}<.05, \mathrm{SE}=.712)$, Oct-4 $(+3.58$-fold $)$, and Nanog (+ 11.58-fold) (Graph 2). Conversely, at higher dosages quinine decreased stemness markers compared to the control after 14 days: Sox2 $(+.51$-fold $)$, cMyc $(+$ .16 -fold), Oct-4 (+ .09-fold, p <.05, SE = .003), Nanog $(+$ .06 -fold, $\mathrm{p}<.05$ ) (figure 2).

\section{Quinine induced TGF $\beta 1$ and Ki67 but decreased BMP7 expression}

Lastly, quinine increases TGF $\beta 1$ across all quinine concentrations - except at IDC $\times 0.01(+9.06$-fold average, $\mathrm{p}<$ $.05, \mathrm{SE}=1.48)$. and decreases BMP7 significantly at IDC $\times$ $0.001(+.12$-fold, $\mathrm{p}<.05, \mathrm{SE}=.001)$. Quinine also increases Ki67 at IDC $\times 0.1(+17.66$-fold, $\mathrm{p}<.05, \mathrm{SE}=.5299)$ (figure 3).

\section{DISCUSSION}

Gluteal fibrosis (GF) is unfortunately quite common in certain areas of Uganda and many other countries and has a significant negative impact on the hip function in affected children and adults. As many as $28 \%$ of visits to a musculoskeletal clinic in the Kumi District of Uganda are related to injection-induced GF (2). Various studies have indicated that over $80 \%$ of children affected by GF had received multiple IM quinine injections prior to the development of the fibrosis (1, 4). Few studies have investigated the microscopic phenomena that leads to the development of this disabling condition. Furthermore, no studies have been conducted investigating the role that antimalarial drugs, such as quinine, play in gluteal fibrosis' development. As such, we investigated a commonly IM administered antimalarial in Uganda, quinine, and found that it plays a key role in causing fibrotic changes to skeletal muscle 


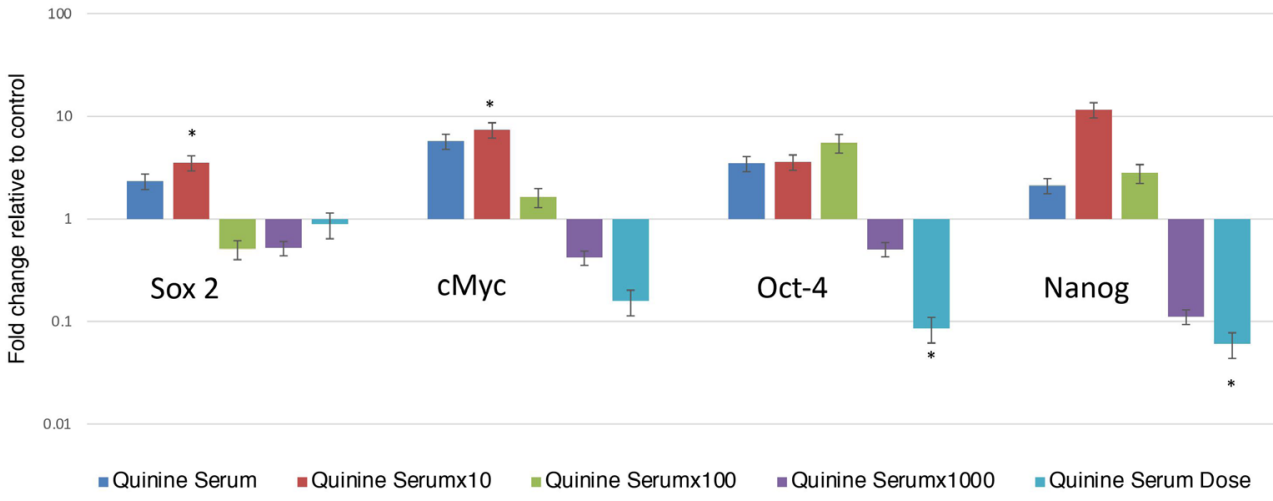

Figure 2. Quinine increases stemness markers at lower doses but decreases those markers at higher doses in FAPs.$$
100
$$

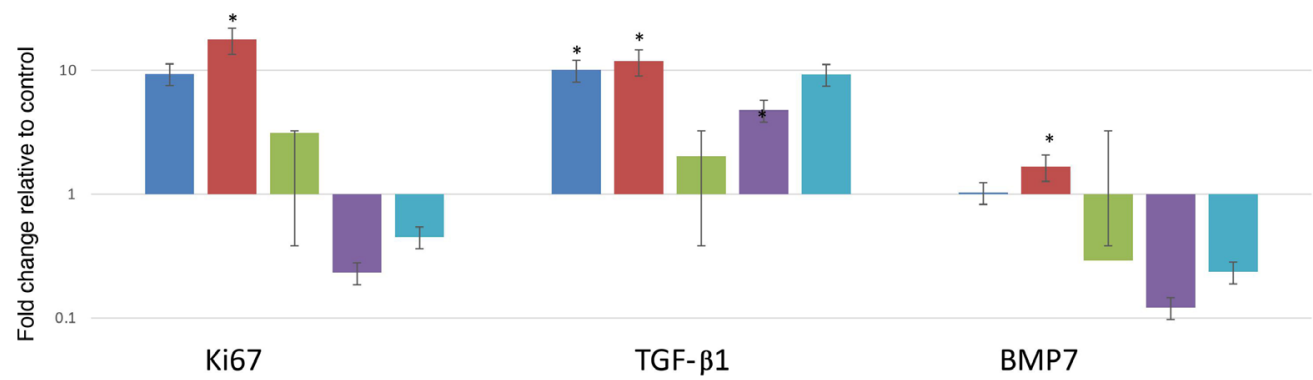

001

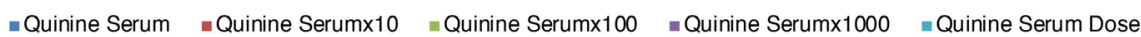

Figure 3. Quinine increases TGF $\beta 1$ and Ki67 expression but decreases BMP7 expression in FAPs.

cells $(1,2,4,5,7-9,11,12)$. Quinine ultimately promotes skeletal muscle fibrotic changes by inducing FAP fibrogenesis and reducing FAP stemness via the TGF- $\beta 1 / \mathrm{BMP} 7$ pathway. Furthermore, our findings suggest that quinine's microscopic effects on human myocytes potentially account for the development of macroscopic gluteal contractures (4-6, 11, 13-16).

Despite decades of studies reporting the presence of, and treatment for GF, little information is available about the cellular mechanisms by which GF develops. Recently, the Transforming Growth Factor- $\beta$ (TGF- $\beta$ ) signaling pathway has been under investigation in relation to its effects on skeletal muscle FAP's. This pathway has been found to play a pivotal role in the development of pathologic fibrosis in various tissues and organs (13-15). Specifically, studies have shown that TGF- $\beta$ is associated with increased fibrosis of injured skeletal muscle by preventing FAP apoptosis (13-15). Furthermore, FAP's have been identified as integral cell precursors for skeletal muscle adipocyte infiltration and fibrosis $(14,15)$. FAP's are integral regulators of satellite cells (skeletal muscle stem cells) in acute injury states. They have been linked to the development of pathologic states within skeletal muscle such as impaired regeneration, fatty infiltration and fibrosis $(14,15)$. We believe that the TGF- $\beta$ pathway is the key regulator of the fibrotic changes seen in skeletal muscle during pathologic injury states, such as GF, due to its role in regulating FAP apoptosis.

The Transforming Growth Factor- $\beta$ (TGF- $\beta$ ) signaling pathway has been identified as a superfamily of polypeptide ligands $(14,15)$. Components of the TGF- $\beta$ superfamily include: TGF- $\beta$-like ligands, bone morphogenetic proteins (BMPs) and activins. The TGF- $\beta$ superfamily of ligands is incredibly important for the proliferation, differentiation and development of skeletal muscle cells $(14,15)$. TGF- $\beta$ 's cellular effects depend on the duration of its activation. In an acute setting, TGF- $\beta$ has been found to assist in skeletal muscle repair $(14,15)$. However, when skeletal muscle is chronically exposed to TGF- $\beta$, pathologic fibrosis develops (14, 15). Similarly, our results demonstrate that chronic activation of the TGF- $\beta$ pathway expression results in pathologic muscle fibrosis seen in FAPs exposed to quinine. Throughout almost every quinine exposed condition, TGF- $\beta 1$ was found to be upregulated and BMP7 downregulated. These results along with the supporting literature on the TGF- $\beta$ pathway's role as a key regulator in skeletal muscle fibrosis leads us to believe that quinine activates this pathway, which in turn leads to pathologic muscle fibrosis.

RT-qPCR confirmed that quinine induces FAP phenotypic changes and increased fibrogenesis as determined by the up-regulation of collagen- 1 , vimentin, and $\alpha \mathrm{SMA}$ expression 
$(11,13,14,16)$. Furthermore, at high dosages quinine downregulates Nanog, Sox2, Oct-4 and cMyc stemness markers, but at lower dosages quinine increases stemness markers in FAPs (16). These results highlight that at lower concentrations quinine has a therapeutic effect on cells by increasing stemness, at higher concentrations, quinine can be especially determinantal to the cells. We postulate that quinine's ability to decrease stemness markers and increase fibrogenic markers is due to the upregulation of TGF- $\beta 1$ and the downregulation of BMP7 based on our RT-qPCR findings $(14,15)$.

Because this is a pilot study, our lab is aware that there are limitations to our initial investigation of this topic. Limitations include that the cells we used were mouse cells, not human muscle cells and therefore they may respond differently than human cells do. Further, this study was not conducted in vivo, and our samples lacked exposure to mechanical stress from syringe administration and fluid infiltration which may also be factors in development of gluteal fibrosis.

In an effort to better understand the etiology of GF we desire to repeat these studies with quinine in comparison to other commonly injected medications $(1-4,7)$. Furthermore, we will explore options for an in vivo mouse model. These approaches will allow us to gain a better understanding of this condition and the factors leading to the development of GF. Further studies to explore the effects of the mechanical act of delivering the injection, the potential role of micro-abscess formation after sub-sterile preparation

\section{REFERENCES}

1. Alves K, Godwin CL, Chen A, Akellot D, Katz JN, Sabatini CS. Gluteal fibrosis, post-injection paralysis, and related injection practices in Uganda: a qualitative analysis. BMC Health Serv Res 2018;18(1):892.

2. Alves K, Penny N, Kobusingye O, Olupot R, Katz JN, Sabatini CS. Paediatric musculoskeletal disease in Kumi District, Uganda: a cross-sectional survey. Int Orthop 2018;42(8):1967-73.

3. Farrar J. Manson's tropical diseases: Expert consult - online and print. 23rd ed. London, England: W B Saunders; 2013:p. 532.

4. McCloskey JR, Chung MK. Quadriceps contracture as a result of multiple intramuscular injection. Am J Dis Child 1977;131(4):416-7.

5. Onimus M, Brunet L, Gaudeuille A, Mapouka AI. Treatment of complications of intramuscular injection of quinine salts in Africa. Med Trop (Mars) 2007;67(3):267-73.

6. Soumah MT, Sylla AI, Toure MR, et al. Quadriceps fibrosis following intramuscular injections into the thigh: apropos of 92 cases at the Ignace Deen Central University Hospital in Conakry. Med Trop (Mars) 2003;63(1):49-52.

7. AnneLoes van Staa AH. Injection practices in the developing world Results and recommendations from field studies in Uganda and Indonesia. WHO Action Programme on Essential Drugs 1996;20:127. and if similar results are seen with other commonly injected medications would be beneficial, as the development of GF may be multi-factorial in nature.

\section{CONCLUSIONS}

Overall, our findings suggest that quinine induces FAP fibrogenesis and reduces FAP stemness. Furthermore, we postulate the TGF- $31 / \mathrm{BMP} 7$ pathway is the underlying mechanism for quinine-induced muscle fibrosis. In a clinical sense, these findings suggest that quinine contributes to the fibrosis of human myocytes by increasing collagen-1, vimentin and $\alpha \mathrm{SMA}$ production in FAPs while also causing FAP's to differentiate into their fibrogenic counterparts $(4,11,13,15,16)$. The microscopic phenomena we found lead us to believe that quinine can potentially contribute to the development of macroscopic gluteal contractures through the same mechanisms $(4,5,6,11,13-16)$. However, these findings do not prove that quinine is solely responsible for gluteal contractures but suggest that quinine may contribute.

Future work is needed to demonstrate how quinine interacts with TGF- $\beta 1$ and BMP7 pathways in FAPs.

\section{CONFLICT OF INTERESTS}

The authors declare that they have no conflict of interests.

8. Waller D, Krishna S, Craddock C, et al. The pharmacokinetic properties of intramuscular quinine in Gambian children with severe falciparum malaria. Trans R Soc Trop Med Hyg 1990;84(4):488-91.

9. World Health Organization. WHO model prescribing information: drugs used in parasitic diseases, 2nd ed. World Health Organization 1995:p 52

10. Wyatt HV. The popularity of injections in the Third World: origins and consequences for poliomyelitis. Soc Sci Med 1984;19(9):911-5.

11. Joe AWB, Yi L, Natarajan A, et al. Muscle injury activates resident fibro/adipogenic progenitors that facilitate myogenesis. Nat Cell Biol 2010;12(2):153-63.

12. Verdier M-C, Bentué-Ferrer D, Tribut O, pour le groupe Suivi Therapeutique Pharmacologique de la Societe Francaise de Pharmacologie et de Therapeutique. Suivi thérapeutique pharmacologique de la quinine. Therapie 2011;66(6):507-16.

13. Arora PD, McCulloch CA. Dependence of collagen remodelling on alpha-smooth muscle actin expression by fibroblasts. J Cell Physiol 1994;159(1):161-75.

14. Desmoulière A, Geinoz A, Gabbiani F, Gabbiani G. Transforming growth factor-beta 1 induces alpha-smooth muscle actin expression in granulation tissue myofibroblasts and 
in quiescent and growing cultured fibroblasts. J Cell Biol 1993;122(1):103-11.

15. Davies MR, Liu X, Lee L, et al. TGF- $\beta$ small molecule inhibitor SB431542 reduces rotator cuff muscle fibrosis and fatty infiltration by promoting fibro/adipogenic progenitor apoptosis. PLoS One 2016;11(5):e0155486.

16. Natarajan A, Lemos DR, Rossi FMV. Fibro/adipogenic progenitors: a double-edged sword in skeletal muscle regeneration. Cell Cycle 2010;9(11):2045-6.

17. Lerkiatbundit S. Stability of quinine dihydrochloride in commonly used intravenous solutions. J Clin Pharm Ther 1993;18(5):343-5.
18. Sabchareon A, Chongsuphajaisiddhi T, Attanath P. Serum quinine concentrations following the initial dose in children with falciparum malaria. Southeast Asian J Trop Med Public Health 1982;13(4):556-62.

19. Padulo J, Oliva F, Frizziero A, Maffulli N. Muscles, Ligaments and Tendons Journal - Basic principles and recommendations in clinical and field Science Research: 2018 update. Muscles Ligaments Tendons J 2018;8(3):305-7.

20. Geraghty RJ, Capes-Davis A, Davis JM, et al. Guidelines for the use of cell lines in biomedical research. Br J Cancer 2014;111(6):1021-46. 